\title{
Pure Membrane, Pseudo Membrane, and Semi Membrane Shell Theories of Hybrid Anisotropic Materials
}

\author{
Chung S. W. ${ }^{1}$, Hong S. G. ${ }^{2}$ and Ju Gi $\mathrm{Su}^{3}$ \\ 1. University of Utah, Salt Lake City, Utah, USA \\ 2. Seoul National University, Seoul, South Korea \\ 3. Yeung Nam University, Tae Gu, South Korea
}

\begin{abstract}
Pure, pseudo and semi membrane theories may sound similar but totally different theories as well as its behavior. The differences are more significant when it comes to hybrid anisotropic materials, namely laminated shell wall thickness. The nomenclatures and classifications have been existed centuries for isotropic material shells since Donnell and Vlasov era. The methods of formulation of the theories are unique and never been used by others except by the authors. Governing differential equations are uniquely formulated for each theory by use of asymptotic expansion method which has never been used by others for isotropic or anisotropic materials. Longitudinal (L) and circumferential ( $\Pi$ or $l$ ) length scale were introduced during the course of asymptotic expansion method and the different theories among membrane theory are apparently classified. Characteristic behaviors of each theory are shown.
\end{abstract}

Key words: Cylindrical shell theory, hybrid anisotropic material, pseudo-membrane.

\section{Introduction}

The membrane theory of shells is simple and has existed for generations since theoretically formulated by Refs. [1-5].

The properties of anisotropic materials are represented by different elastic coefficients and different cross-ply angles. The cross-ply angle, $y$, is the angle between major elastic axis of the material and reference axis ( Figs. 1 and 2).

According to the exact three-dimensional theory of elasticity, a shell element is considered as a volume element. All possible stresses and strains are assumed to exist, and no simplifying assumptions are allowed in the formulation of the theory. We therefore allow for six stress components, six strain components and three displacements as indicated in the following relation:

$$
\text { STRESSES }=\text { [ELASTIC COEFFICIENTS }] \times
$$

Corresponding author: Chung S. W., all professors, research fields: mechanics of materials.

\section{[STRAINS]}

There are a total of fifteen unknowns to solve for in a three dimensional elasticity problem. On the other hand, the equilibrium equations and strain displacement equations can be obtained for a volume element and six generalized elasticity equations can be used. A total of fifteen equations can thus be formulated, and it is basically possible to set up a solution for a three-dimensional elasticity problem.

In the first part of this article, the asymptotic expansion and integration method is used to reduce the exact three-dimensional elasticity theory for a non-homogeneous, anisotropic cylindrical shell to approximate theories. The analysis is made such that it is valid for materials which are non-homogeneous to the extent that their mechanical properties are allowed to vary with the thickness coordinate. The derivation of the theories is accomplished by first introducing the shell dimensions and, as yet unspecified characteristic, length scales via changes in the independent variables. 


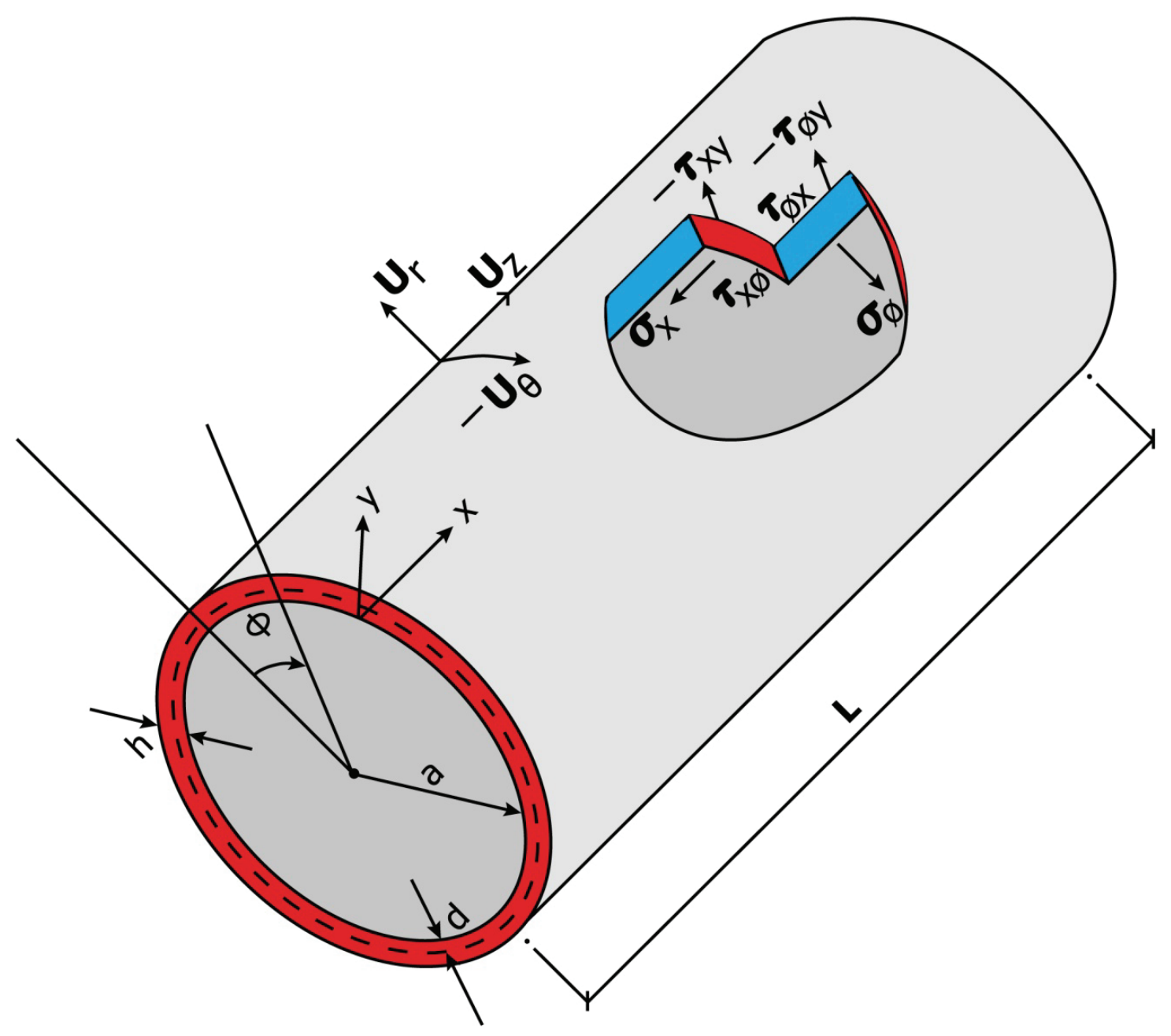

\section{CYLINDRICAL COORDINATE}

Fig. 1 Dimensions, deformations and stresses of the cylindrical shell.

Next, the dimensionless stresses and displacements are expanded asymptotically by using the thinness of the shell as the expansion parameter. A choice of characteristic length scales is then made and, corresponding to different combination of these length scales, different sequences of systems of differential equations are obtained. Subsequent integration over the thickness and satisfaction of the boundary conditions yields the desired equations governing the formulation of the first approximation stress states of a non-homogeneous anisotropic cylindrical shell.

\section{Formulation of Cylindrical Shell Theory of Anisotropic Materials}

Consider a non-homogeneous, anisotropic volume element of a cylindrical body with longitudinal, circumferential (angular) and radial coordinates being noted as $z, \theta, r$, respectively, and subjected to all possible stresses and strains ( Fig. 1).

Here, $a$ is the inner radius, $h$ the thickness and $L$ the length.

$a$ : inside radius of cylindrical shell; 


$$
\begin{array}{ll}
z \text {; Longitudinal, } & x=\frac{z}{L} \\
\theta \text {; Circumferential, } & \phi=\frac{a}{\beta}=\frac{\theta a}{\Pi} \\
r \text {; Radial, } & y=\frac{r-a}{h}
\end{array}
$$

\section{L, $\Pi$; Longitudinal and circumferential} length scales

a ; I.D. of cylinder

h ; Total thickness of shell wall

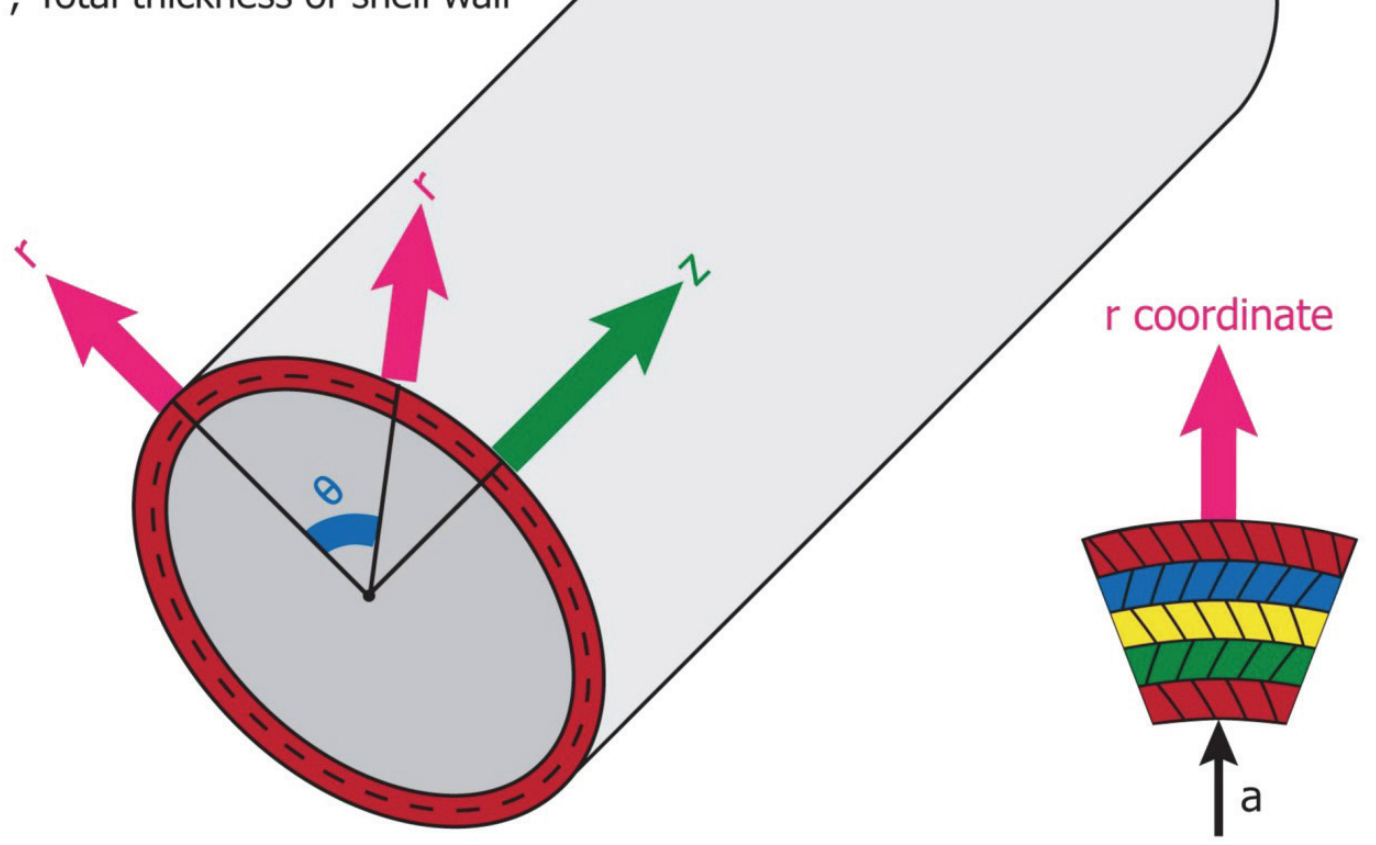

Fig. 2 Details of the coordinate system.

$h$ : total thickness of the shell wall;

$d$ : distance (thickness) from inside radius to mechanical neutral surface;

$S_{\mathrm{i}}$ : radius of each layer of wall $(i=1,2,3 \ldots$ to the number of layer);

$L$ : longitudinal length scale to be defined, also actual length of the cylindrical shell;

$\Pi$ : circumferential length scale of cylindrical shell to be defined;

$E_{i}$ : Young's moduli in $i$ direction;

$G_{i j}$ : shear moduli in $i-j$ Face;

$S_{i j}$ : compliance matrix of materials of each layer;

$r$ : radial coordinate;

$\Pi$ : circumferential length scale to be defined; $\gamma$ : angle of fiber orientation;

$\sigma:$ normal stresses;

$\varepsilon:$ normal strains;

$z, \theta, r$ : generalized coordinates in longitudinal, circumferential and radial directions respectively;

$\tau$ : shear stresses;

$\varepsilon_{i j}$ : shear strains in $i-j$ face;

$\lambda$ : shell thickness/inside radius $(\mathrm{h} / \mathrm{a})$;

$C_{i j}$ : elastic moduli in general;

$X, \varphi, Y$ : non dimensional coordinate system in longitudinal, circumferential and radial directions respectively.

Assuming that the deformations are sufficiently small so that linear elasticity theory is valid, the 
following equations govern the problem:

$$
\begin{gathered}
\left(r \tau_{r z}\right)_{,_{r}}+\tau_{\theta z, \theta}+\left(r \sigma_{z}\right),_{z}=0 \\
\left(r \tau_{r \theta}\right)_{r}+\sigma_{\theta, \theta}+\left(r \tau_{\theta z}\right),_{r_{z}}+\tau_{\theta z}=0 \\
\left(r \sigma_{r}\right),_{r}+\tau_{r \theta, \theta}+\left(r \tau_{r z}\right),_{z}-\sigma_{\theta}=0 \\
u_{z, z}=S_{11} \sigma_{z}+S_{12} \sigma_{\theta}+S_{13} \sigma_{r}+S_{14} \tau_{r \theta}+S_{15} \tau_{r z}+S_{16} \tau_{\theta z} \\
\frac{1}{r}\left(u_{\theta, \theta}+u_{r}\right)=S_{12} \sigma_{z}+S_{22} \sigma_{\theta}+\cdots+S_{26} \tau_{\theta z} \\
u_{r, z}=S_{13} \sigma_{z}+\cdots+S_{36} \tau_{\theta z} \\
\frac{1}{r} u_{r, \theta}+u_{\theta z}-\frac{1}{r} u_{\theta}=S_{14} \sigma_{z}+\cdots+S_{16} \tau_{\theta z} \\
u_{z, r}+u_{r, z}=S_{15} \sigma_{z}+\cdots+S_{36} \tau_{\theta z} \\
u_{\theta, z}+\frac{1}{r} u_{z, \theta}=S_{16} \sigma_{z}+\cdots+S_{66} \tau_{\theta z}
\end{gathered}
$$

In Eq. (1), there are equilibrium equations and complete anisotropy of the material which is allowed for and there are thus 21 independent material constants. We are not allowed to illuminate any of those components, since the material properties are dependent on the manufactures set up and different gravity environment in case of aerospace vehicles. Also the compliance matrix is symmetric, $S_{i j}$ is the same as $S_{j i}$, and the components can be expressed in terms of engineering constants as follows:

$$
\begin{aligned}
& S_{i i}=\frac{1}{E_{i}},(i=1,3) \\
& S_{i j}=\frac{-v_{i j}}{E_{i}},(i=1,2, j=2,3, i \neq j) \\
& S_{44}=\frac{1}{G_{23}} \\
& S_{55}=\frac{1}{G_{13}} \\
& S_{66}=\frac{1}{G_{12}}
\end{aligned}
$$

In Eq. (2) the $E_{i}$ 's are the Young's moduli in tension along the $i$-direction and Eq. (2) implies anisotropic property of the material only, material to be non-homogeneous, different properties of each layer of the shell, we will allow the material property variation in the radial direction as follows:

$$
S_{i j}=S_{i j}(r)
$$

Eq. (3) is unique and different from most of conventional theories, including Ref. [6], which input the engineering constants artificially from the beginning, while we take the existence and magnitude of components only by approximation theory of the asymptotic expansion.

The principal material axes in general do not coincide with the body axes with respect to material axes specified, then the properties with respect to the body axes are given by the following transformation equations:

$$
\begin{gathered}
S_{11}=S_{11}^{\prime} \cos ^{4} \gamma+\left(2 S_{12}^{\prime}+S_{66}^{\prime}\right) \sin ^{2} \gamma \cos ^{2} \gamma+S_{22}^{\prime} \sin ^{4} \gamma \\
+\left(S_{16}^{\prime} \cos ^{2} \gamma+S_{26}^{\prime} \sin ^{2} \gamma\right) \sin 2 \gamma, \\
S_{22}=S_{11}^{\prime} \cos ^{4} \gamma+\left(2 S_{12}^{\prime}+S_{66}^{\prime}\right) \sin ^{2} \gamma \cos ^{2} \gamma+S_{22}^{\prime} \cos ^{4} \gamma \\
\quad-\left(S_{16}^{\prime} \sin ^{2} \gamma+S_{26}^{\prime} \cos ^{2} \gamma\right) \sin 2 \gamma, \\
S_{12}=S_{12}^{\prime}+\left(S_{11}^{\prime}+S_{22}^{\prime}-2 S_{12}^{\prime}-S_{66}^{\prime}\right) \sin ^{2} \gamma \cos ^{2} \gamma \\
+\frac{1}{2}\left(S_{26}^{\prime}-S_{16}^{\prime}\right) \sin 2 \gamma \cos 2 \gamma,
\end{gathered}
$$




$$
\begin{aligned}
S_{66}= & S_{66}^{\prime}+4\left(S_{11}^{\prime}+S_{22}^{\prime}-2 S_{12}^{\prime}-S_{66}^{\prime}\right) \sin ^{2} \gamma \cos ^{2} \gamma+2\left(S_{26}^{\prime}+S_{16}^{\prime}\right) \sin 2 \gamma \cos 2 \gamma, \\
S_{16}= & {\left[S_{22}^{\prime} \sin ^{2} \gamma-S_{11}^{\prime} \cos ^{2} \gamma+\frac{1}{2}\left(2 S_{12}^{\prime}+S_{66}^{\prime}\right) \cos 2 \gamma\right] \sin 2 \gamma+S_{16}^{\prime} \cos ^{2} \gamma\left(\cos ^{2} \gamma-3 \sin ^{2} \gamma\right) } \\
& +S_{26}^{\prime} \sin ^{2} \gamma\left(3 \cos ^{2} \gamma-\sin ^{2} \gamma\right), \\
S_{26}= & {\left[S_{22}^{\prime} \cos ^{2} \gamma-S_{11}^{\prime} \sin ^{2} \gamma+\frac{1}{2}\left(2 S_{12}^{\prime}+S_{66}^{\prime}\right) \cos 2 \gamma\right] \sin 2 \gamma } \\
& +S_{16}^{\prime} \sin ^{2} \gamma\left(3 \cos ^{2} \gamma-\sin ^{2} \gamma\right)+S_{26}^{\prime} \cos ^{2} \gamma\left(\cos ^{2} \gamma-3 \sin ^{2} \gamma\right) .
\end{aligned}
$$

where $\gamma$ is the angle of anisotropic orientation between the original longitudinal coordinate $z$ axes.

The shell is subjected to a uniformly distributed tensile force, then the boundary conditions are as follows:

$$
\begin{aligned}
& \sigma_{r}=\tau_{r \theta}=\tau_{r z}=0 \quad(r=a) \\
& \sigma_{r}=\rho(\theta, z), \tau_{r \theta}=\tau_{r z}=0 \quad(r=a+h)
\end{aligned}
$$

We will find it convenient to work with stress resultants rather than the stresses themselves. These stress resultants, which are forces and moments per unit length, are obtained by integrating with respect to the thickness coordinate. They are:

$$
\begin{aligned}
& N_{z}=\int_{a}^{a+h} \sigma_{z}\left[1+\frac{r-a-d}{a+d}\right] d r \\
& N_{\theta}=\int_{a}^{a+h} \sigma_{\theta} d r \\
& N_{\theta z}=\int_{a}^{a+h} \tau_{\theta z} d r \\
& N_{z \theta}=\int_{a}^{a+h} \tau_{\theta z}\left[1+\frac{r-a-d}{a+d}\right] d r \\
& M_{\theta}=\int_{a}^{a+h} a_{\theta}[r-a-d] d r \\
& M_{z}=\int_{a}^{a+h} a_{z}\left[\frac{r-a-d}{a+d}\right] r d r \\
& M_{\theta z}=\int_{a}^{a+h} \tau_{\theta z}[r-a-d] d r \\
& M_{\theta z}=\int_{a}^{a+h} \tau_{\theta z}\left[\frac{r-a-d}{a+d}\right] r d r
\end{aligned}
$$

In Eq. (6), $a$, denotes the inner radius of the cylindrical shell and, $d$, the distance from the inner surface to the reference surface where the stress resultants are defined.

\section{Formulation of a Boundary Layer Theory}

As a first step to integrate Eq. (6), we make them non-dimensionalized coordinates as follows:

$$
X=z / L, \quad Y=(r-a) / h, \quad \varphi=\theta / \beta
$$

are quantities which are to be determined later.

The parameters $L$ and $\Pi$ are thus seen to be characteristic length scales for changes of the stresses and displacements in the axial and circumferential directions, respectively where $\lambda$ is the thin shell parameter defined as

$$
\lambda=h / a
$$

The parameter $\lambda$ is representative of the thinness of the cylindrical shell.

$$
\lambda<<1
$$

The first system of equations of "thin shell" theory and we will call it the first approximation system. We can however obtain stresses and displacements of each layer of thickness coordinate, that can be an advantage of the procedure among others. In the following section, the thin shell theories for different combinations of length scales will be derived.

\section{Formulation of Pure Membrane Theory}

(Associated with characteristic length scales, $a$ ) As we observed, the shell geometry is an important factor for the formulation of theories.

The basic geometry of cylindrical shells is the 
longitudinal length $L$, inside radius $a$, total wall thickness $h$ and the distance from inner surface to a desired surface, $d$. We are interested here in deriving the shell theory associated with the case where the axial and circumferential length scales are both equal to the inner radius of the cylinder, $a$, as follows:

$$
L=a, \quad \Pi=a
$$

Here we adopt the concept of characteristic length scale first developed by Calladine in the Eq. (3.8) in Ref. [7].

The reason for taking the length scales, $a$, is the longest practical dimension of the shell and we are interested in developing membrane type theory which requires longer than the bending characteristic influential length according to the classical theory of isotropic materials.

Substituting these length scales into the three-dimensional elasticity Eq. (1) and the asymptotic expansions the following equations representing the first approximation theory of the problem result upon use of the procedure outlined earlier

$$
\begin{gathered}
v_{r, y}^{(0)}=0 \\
v_{z, y}^{(0)}=0 \\
v_{\theta, y}^{(0)}=0 \\
v_{z, x}^{(0)}=s_{11}^{(0)} t_{z}^{(0)}+s_{12}^{(0)} t_{\theta}^{(0)}+s_{16}^{(0)} t_{\theta z}^{(0)} \\
v_{\theta, \phi}^{(0)}+v_{r}^{(0)}=s_{21}^{(0)} t_{z}^{(0)}+s_{22}^{(0)} t_{\theta}^{(0)}+s_{26}^{(0)} t_{\theta z}^{(0)} \\
v_{\theta, x}^{(0)}+v_{z, \phi}^{(0)}=s_{61}^{(0)} t_{z}^{(0)}+s_{62}^{(0)} t_{\theta}^{(0)}+s_{66}^{(0)} t_{\theta z}^{(0)} \\
t_{r z, y}^{(2)}+t_{\theta z, \phi}^{(0)}+t_{z, x}^{(0)}=0 \\
t_{r \theta, y}^{(2)}+t_{\theta, \phi}^{(0)}+t_{\theta z, x}^{(0)}=0 \\
t_{r, y}^{(2)}+t_{\theta}^{(0)}=0
\end{gathered}
$$

The superscripts indicate the leading term in each of the above equations and represent the relative of magnitude of the displacements and stresses. These orders of magnitude result from the intention to obtain a system of equations which is inferable with respect to the thickness coordinate $y$ in a step-by-step manner with the following additional reasoning:

(a) The dominant stress state in thin shell theory is the in-plane stress state. These stresses should be of the same order of magnitude.

(b) The order of the displacements is chosen so that the product of the in-plane strains and the elastic moduli is of the same order of magnitude as the in-plane stresses.

(c) The choice for the transverse stresses arises from the fact that they should contribute terms of the same magnitude in the equilibrium. Integration of the first three equations of Eq. (10) with respect to $y$ yields

$$
\begin{aligned}
& v_{r}^{(0)}=v_{r}^{(0)}(x, \phi) \\
& v_{z}^{(0)}=v_{z}^{(0)}(x, \phi) \\
& v_{\theta}^{(0)}=v_{\theta}^{(0)}(x, \phi)
\end{aligned}
$$

where $, v_{r}, v_{z}, v_{\theta}$ are the displacements in $r, z$ and $\theta$ directions respectively.

The middle three equations of Eq. (10) can be solved for the in-plane stresses as follows:

$$
\left\{\begin{array}{l}
t_{z}^{(0)} \\
t_{\theta}^{(0)} \\
t_{\theta z}^{(0)}
\end{array}\right\}=[C]\left\{\begin{array}{c}
\varepsilon_{1} \\
\varepsilon_{2} \\
\varepsilon_{12}
\end{array}\right\}
$$

Here, $\mathrm{C}(i, j=1,2,3)$ are the components of a symmetric matrix given by

$$
C=\left[\begin{array}{lll}
s_{11}^{(0)} & s_{12}^{(0)} & s_{16}^{(0)} \\
s_{12}^{(0)} & s_{22}^{(0)} & s_{26}^{(0)} \\
s_{16}^{(0)} & s_{26}^{(0)} & s_{66}^{(0)}
\end{array}\right]^{-1}
$$

From Eqs. (12) and (13) we can obtain strains as follows:

$$
\left\{\begin{array}{c}
\varepsilon_{1} \\
\varepsilon_{2} \\
\varepsilon_{12}
\end{array}\right\}=\left\{\begin{array}{c}
v_{z, x}^{(0)} \\
v_{\theta, \phi}^{(0)}+v_{r}^{(0)} \\
v_{\theta, x}^{(0)}+v_{r, \phi}^{(0)}
\end{array}\right\}
$$

On substituting the first approximation in-plane stress-strain relations into the last three equations of Eq. (10) and integrating with respect to $y$, we obtain: 


$$
\begin{aligned}
t_{r z}=T_{r z}(x, \phi)- & {\left[A_{13} V_{z, x \phi}+A_{23}\left(V_{\theta, \phi \phi}+V_{r, \phi}\right)+A_{33}\left(V_{\theta, x \phi}+V_{z, \phi \phi}\right)\right] } \\
& -\left[A_{11} V_{z, x x}+A_{12}\left(V_{\theta, x \phi}+V_{r, x}\right)+A_{13}\left(V_{\theta, x x}+V_{z, x \phi}\right)\right] \\
t_{r \theta}= & T_{r \theta}(x, \phi)-\left[A_{12} V_{z, x \phi}+A_{22}\left(V_{\theta, \phi \phi}+V_{r, \phi}\right)+A_{23}\left(V_{\theta, x \phi}+V_{z, \phi \phi}\right)\right] \\
& -\left[A_{13} V_{z, x x}+A_{23}\left(V_{\theta, x \phi}+V_{r, x}\right)+A_{33}\left(V_{\theta, x x}+V_{z, x \phi}\right)\right] \\
t_{r}= & T_{r}+A_{12} V_{z, x}+A_{22}\left(V_{\theta, \phi}+V_{r}\right)+A_{23}\left(V_{\theta, x}+V_{z, \phi}\right)
\end{aligned}
$$

where $T_{r z}, T_{r \theta}, T_{r}$ are the transverse stress components of the $r=0$ surface and

$$
A_{i j}=\int_{0}^{y} C_{i j} d \rho
$$

In Eq. (14) and in what is to follow, the superscripts on the displacements have been dropped.

Boundary conditions are to be satisfied by each term of asymptotic expansions. This yields

$$
\begin{aligned}
& t_{r z}^{(2)}=t_{r \theta}^{(2)}=t_{r}^{(2)}=0 \quad(y=0) \\
& t_{r z}^{(2)}=t_{r \theta}^{(2)}=0, \quad t_{r}^{(2)}=p^{*} \quad(y=0)
\end{aligned}
$$

Here, $p^{*}$ is a dimensionless pressure defined by

$$
p^{*}=p /(\sigma \lambda)
$$

Satisfaction of Eq. (17) by Eq. (15) yields

$$
T_{r z}=T_{r \theta}=T_{r}=0
$$

and the following three differential equations for displacements $V z, V_{\theta}$ and $V r$,

$$
\begin{aligned}
& \underline{A}_{13} V_{z, x \phi}+\underline{A}_{23}\left(V_{\theta, \phi \phi}+V_{r, \phi}\right)+\underline{A}_{33}\left(V_{\theta, x \phi}+V_{z, \phi \phi}\right) \\
& +\underline{A}_{11} V_{z, x x}+\underline{A}_{12}\left(V_{\theta, x \phi}+V_{r, x}\right)+\underline{A}_{13}\left(V_{\theta, x x}+V_{z, x \phi}\right)=0 \\
& \underline{A}_{12} V_{z, x \phi}+\underline{A}_{22}\left(V_{\theta, \phi \phi}+V_{r, \phi}\right)+\underline{A}_{23}\left(V_{\theta, x \phi}+V_{z, \phi \phi}\right) \\
& +\underline{A}_{13} V_{z, x x}+\underline{A}_{23}\left(V_{\theta, x \phi}+V_{r, x}\right)+\underline{A}_{33}\left(V_{\theta, x x}+V_{z, x \phi}\right)=0 \\
& \underline{A}_{12} V_{z, x}+\underline{A}_{22}\left(V_{\theta, \phi}+V_{r}\right)+\underline{A}_{23}\left(V_{\theta, x}+V_{z, \phi}\right)=p^{*}
\end{aligned}
$$

In the above equations

$$
\underline{A}_{i j}=A_{i j}^{(1)}
$$

To obtain the appropriate expressions for the stress resultants we first non-dimensionalize those defined by Eq. (9) as follows:

$$
\bar{N}=\frac{N}{\sigma \cdot \lambda \cdot \mathrm{a}}, \quad \bar{M}=\frac{M}{\sigma \cdot \lambda^{2} \cdot \mathrm{a}^{2}}
$$

where $N$ and $M$ are the generic symbols for the force and moment stress resultants, respectively. Note that to non-dimensionalize the force and moment, we divide by same unit of force and moment per unit strip of the shell surface, which is of pound/inch or kilogram/centimeter for force and pound-inch/inch or kilogram-centimeter/centimeter respectively. Assuming it to be possible, we now asymptotically expand each of the dimensionless stress resultants in a power series in $1 / 2$,

$$
\begin{aligned}
& \bar{N}=\sum_{m=0}^{M} N^{(m)}(x, \phi) \cdot \lambda^{m / 2} \\
& \bar{M}=\sum_{m=0}^{M} M^{(m)}(x, \phi) \cdot \lambda^{m / 2}
\end{aligned}
$$

where $N(m)$ and $M(m)$ are of the order unity.

\section{Pseudo-Membrane Phenomena}

We are now interested in a formulation of equations to be able to obtain all the stress resultants due to membrane and bending actions.

On substitution of Eqs. (21) and (22) and the results for in-plane stresses Eq. (15) into Eq. (9) and equating terms of like powers in $1 / 2$ on each side of the equations, we obtain the following expressions for the first approximation stress resultants:

$$
\left.\left\{\begin{array}{l}
N_{z} \\
N_{\theta} \\
N_{z \theta} \\
N_{\theta z} \\
M_{z} \\
M_{\theta} \\
M_{z \theta} \\
M_{\theta z}
\end{array}\right\}=[\overline{\bar{A}}] \overline{\bar{B}}\right]\left\{\begin{array}{c}
\varepsilon_{1} \\
\varepsilon_{2} \\
\varepsilon_{12}
\end{array}\right\}
$$


where the superscript zero has been omitted and $B$ is defined as follows:

$$
B_{i j}=\int_{0}^{y} C_{i j}(\rho) d \rho, \quad \underline{B}_{i j}=B_{i j}(1)
$$

and submatrices $[\mathrm{A}]$ and $[\mathrm{B}]$ are given by

$$
\left.\begin{array}{rl}
{[\bar{A}]} & =\left[\begin{array}{c}
\frac{1}{1+d / a}\left[\underline{A}_{11}, \underline{A}_{12}, \underline{A}_{13}\right] \\
\underline{A}_{21}, \underline{A}_{22}, \underline{A}_{23} \\
\frac{1}{1+d / a}\left[\underline{A}_{31}, \underline{A}_{32}, \underline{A}_{33}\right] \\
\underline{A}_{31}, \underline{A}_{32}, \underline{A}_{33}
\end{array}\right] \\
{[\bar{B}]=\left[\begin{array}{c}
\frac{1}{1+d / a}\left[\left(-\frac{d}{h} \underline{A}_{11}+\underline{B}_{11}\right),\left(-\frac{d}{h} \underline{A}_{12}+\underline{B}_{12}\right),\left(-\frac{d}{h} \underline{A}_{13}+\underline{B}_{13}\right)\right] \\
\frac{1}{1+d / a}\left[\left(-\frac{d}{h} \underline{A}_{21}+\underline{B}_{21}\right),\left(-\frac{d}{h} \underline{A}_{22}+\underline{B}_{22}\right),\left(-\frac{d}{h} \underline{A}_{23}+\underline{B}_{23}\right)\right. \\
\left.\left(-\frac{d}{h} \underline{A}_{31}+\underline{B}_{31}\right),\left(-\frac{d}{h} \underline{A}_{32}+\underline{B}_{32}\right),\left(-\frac{d}{h} \underline{A}_{33}+\underline{B}_{33}\right)\right]
\end{array}\right]}
\end{array}\right]
$$

Note that $d / a$ can be written as

$$
d / a=\lambda(d / h)
$$

From the results obtained above, we characterize the theory as follows:

(a) The approach that this research took, the asymptotic integration, for deriving shell equations is capable of obtaining all stress components, including the transverse components.

(b) The first three equations of Eq. (9) result from the relations for the transverse strains. The variation with respect to $y$ is zero, as shown in the displacements Eq. (23) which are independent of $y$. The strain components of any point y off $r=0$ surface are thus equal to those of $r=0$ surface, similar to classical membrane theory.

(c) The stress components vary with $y$ because as the $y C_{i j}$, and the $A_{i j}$ are functions of $\%$

(d) Eq. (23) shows that moment stress resultants are produced due to the non-homogeneity of the material.

For an isotropic and homogeneous material, $C_{i j}$ are constants and $d / h=1 / 2$.

$$
\underline{B}_{i j}=\frac{1}{2} C_{i j}=\frac{1}{2} \underline{A}_{i j}
$$

On substituting this result into Eq. (25), it is seen that submatrix B is equal to zero and that Eq. (27) becomes those of the classical membrane theory of shell (zero moment resultants). In the case of hybrid anisotropic materials, it is very rare to satisfy all the components of the submatrix [B] to be equal to zero at the same time. In another way of observation, it is unavoidable to associate with some bending moments in addition to pure membrane forces for laminated anisotropic shell walls, as clearly shown in Fig. 5, the blue colored curves are the additional contribution to the pure membrane deformation. Therefore, the analysis is named pseudo-membrane theory. It is 
different from the long effective length of Vlasov's semi membrane theory or the short effective length of Donnell's theory $[2,5,8,9]$.

\section{Formulation of Semi-membrane Theory}

The cylindrical shell theory that we are concerned among the three classifications of above is very long effective length shell which is well compared to Donnell-Vlasov theory. As shown in Fig. 4, the edge effective zone due to the prescribed edge boundary condition represented by curved pattern is limited within close distance from the end and after the zone the deformation is nearly linear with respect to the longitudinal axis, which is very close to membrane analysis. It is more distinct for a cylindrical shell of one end fixed or hinged boundary condition and the other free open. That is semi-membrane status, we will now mathematically formulate the governing equations.

According to Refs. [7, 10-12] respectively, we pick and choose the longitudinal $(L)$ and circumferential $(\Pi)$ length scales as follows.

$$
\mathrm{L}=\mathrm{a}(\mathrm{a} / \mathrm{h})^{1 / 2}, \quad \Pi=\mathbf{a}
$$

We can then obtain the following equations.

Stress-displacement relations:

$$
\begin{aligned}
& v_{r, y}=\lambda\left[\bar{S}_{31} t_{z}+\bar{S}_{32} t_{\theta}+\bar{S}_{33} t_{r}+\bar{S}_{34} t_{r \theta}+\bar{s}_{35} t_{r z}+\bar{S}_{36} t_{\theta z}\right] \\
& v_{z, y}+\lambda^{3 / 2} v_{r, x}=\lambda\left[\bar{S}_{51} t z+\bar{s}_{52} t_{\theta}+\bar{s}_{53} t_{r}+\bar{s}_{54} t_{r \theta}+\bar{s}_{55} t_{r z}+\bar{s}_{56} t_{\theta z}\right] \\
& \lambda v_{r, \phi}+(1+\lambda y) v_{\theta, y}-\lambda v_{\theta} \\
& =\lambda(1+\lambda y)\left[\bar{S}_{41}{ }_{z}+\bar{S}_{42} t_{\theta}+\bar{S}_{43} t_{r}+\bar{S}_{44} t_{r \theta}+\bar{s}_{45}{ }^{t} r z+\bar{S}_{46}{ }^{t} \theta z\right] \\
& \lambda^{1 / 2} v_{z, x}=\bar{s}_{11} t_{z}+\bar{s}_{12} t_{\theta}+\bar{s}_{13} t_{r}+\bar{s}_{14} t_{r_{\theta}}+\bar{s}_{15} t_{r z}+\bar{s}_{16} t_{\theta z}
\end{aligned}
$$

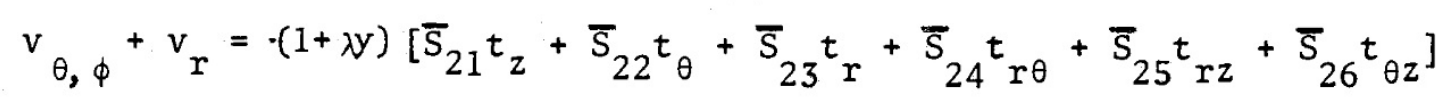

$$
\begin{aligned}
& \lambda^{1 / 2}(1+\lambda y) v_{\theta, x}+v_{z, \phi}
\end{aligned}
$$

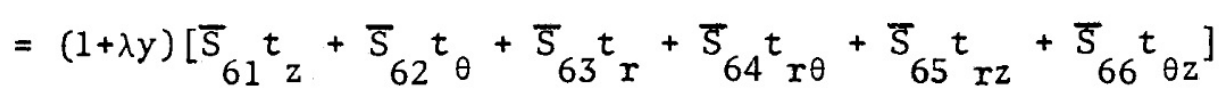

Equilibrium equations:

$$
\begin{aligned}
& {\left[t_{r z}(1+\lambda y)\right]_{y}+\lambda t_{\theta z, \phi}+\lambda^{3 / 2}(1+\lambda y) t_{z, x}=0} \\
& {\left[t_{r \theta}(1+\lambda y)\right]_{y}+\lambda t_{\theta, \phi}+\lambda t_{r \theta}+\lambda^{3 / 2}(1+\lambda y) t_{\theta_{z, x}}=0} \\
& {\left[t_{r}(1+\lambda y)\right]_{y}+\lambda t_{r \theta, \phi}+\lambda^{3 / 2}(1+\lambda y) t_{r z, x}-\lambda t_{\theta}=0}
\end{aligned}
$$


By going through the first approximation procedure of previous formulation as explained in Refs. [12-14], we found the following phenomena.

Transverse strains: the first three equations of Eq. (5) are zero and all displacements shown in Eq. (6) independent of thickness coordinate, $r$, which means it is only membrane state.

In-plane circumferential and shear strains: the longitudinal strain is represented by the combination of longitudinal and circumferential stresses $\left(t_{z}\right.$ and $\left.t_{\theta}\right)$.

In-plane shear stresses: $t_{r z}$ does not appear in the first approximation theory.

We now complete the second approximation theory of the asymptotic expansion and integration procedure, which can be shown as follows:

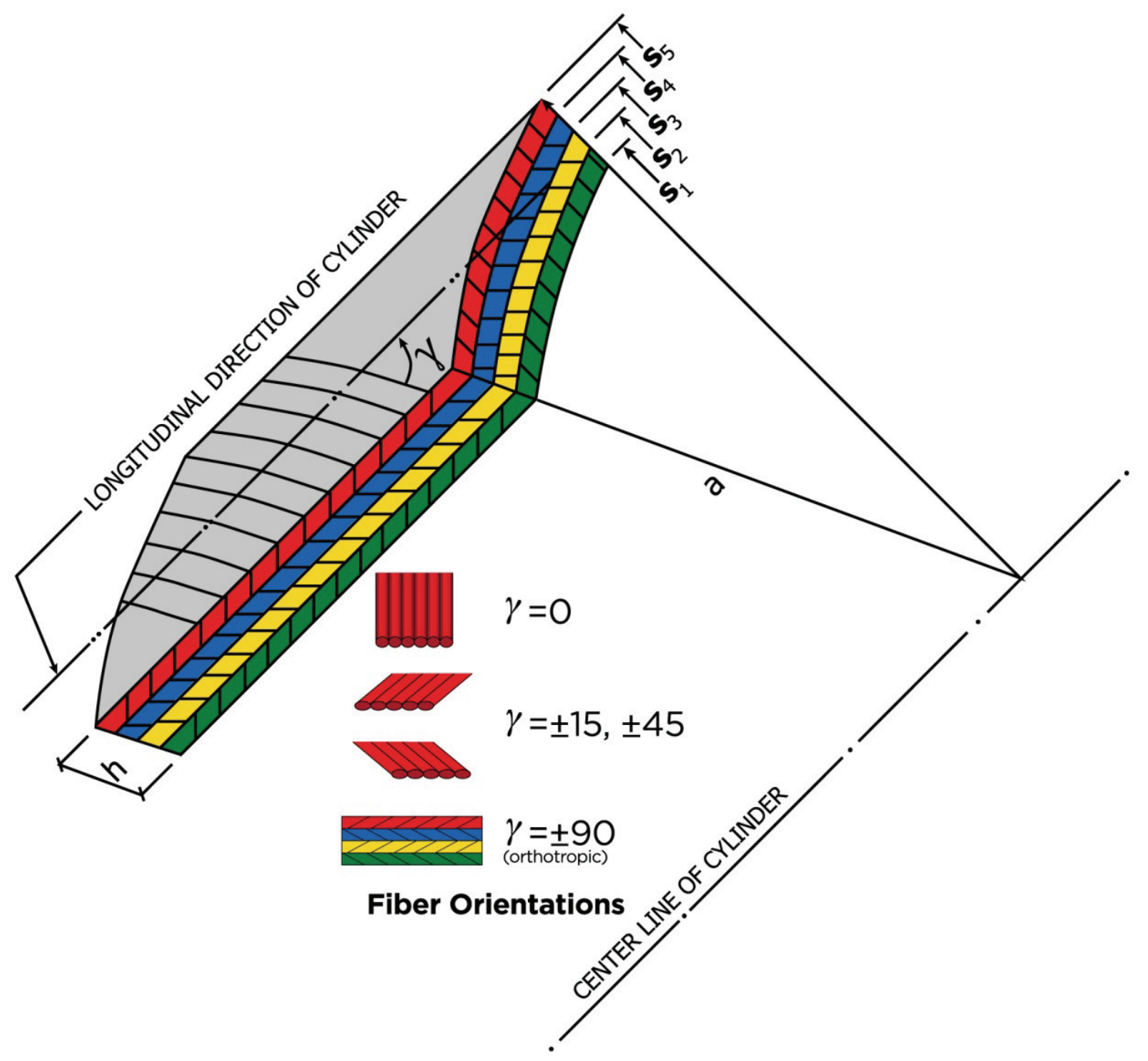

Fig. 3 A laminated cylindrical shell, material orientation $\gamma$. 
Pure Membrane, Pseudo Membrane, and Semi Membrane Shell Theories of Hybrid Anisotropic Materials 131

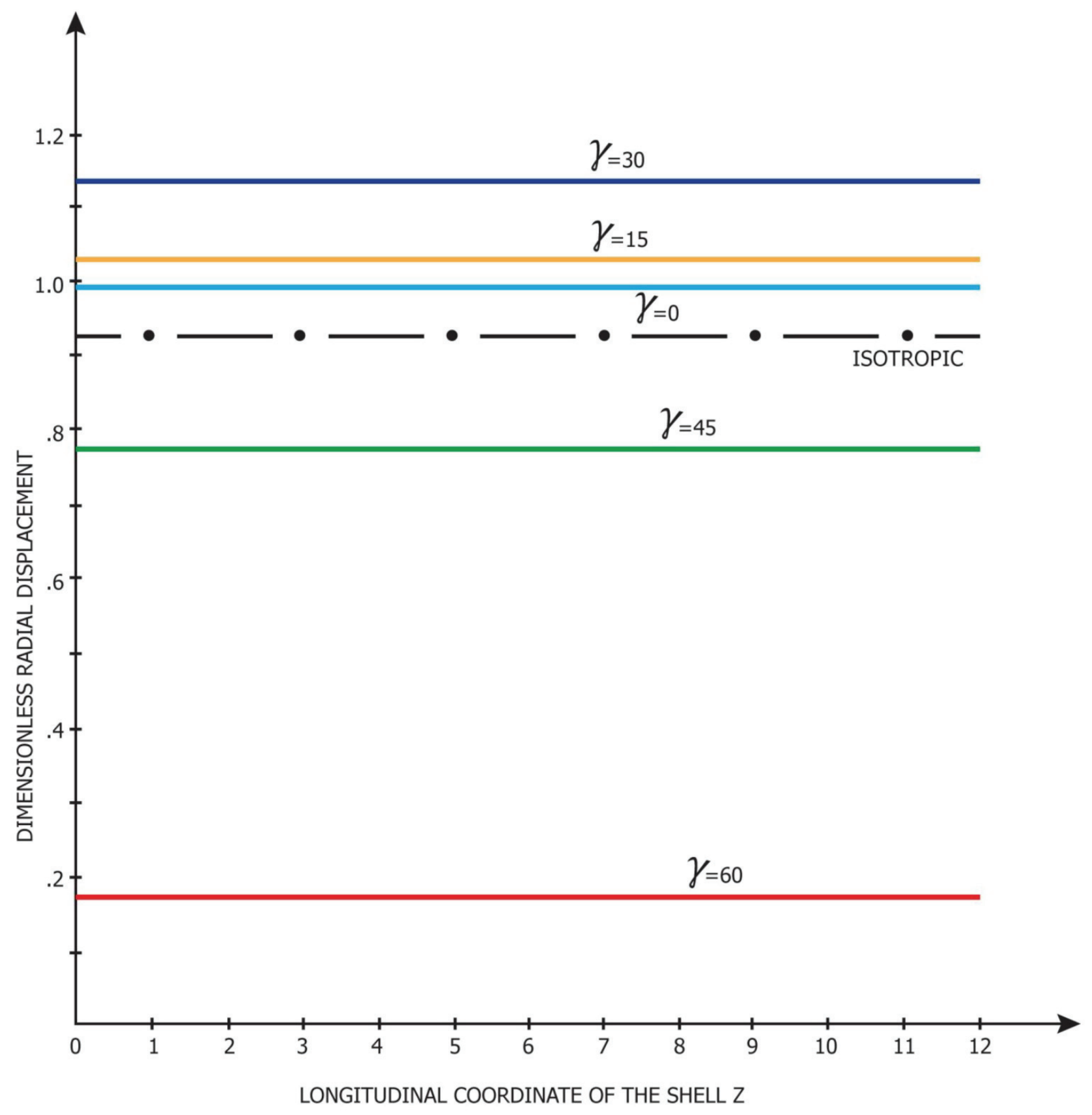

RADIAL DISPLACEMENT OF THE THEORY ASSOCIATED WITH LENGTH SCALES a

Fig. 4 Non-dimensionalized radial displacement of pure theory. 


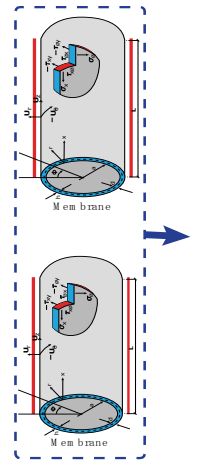

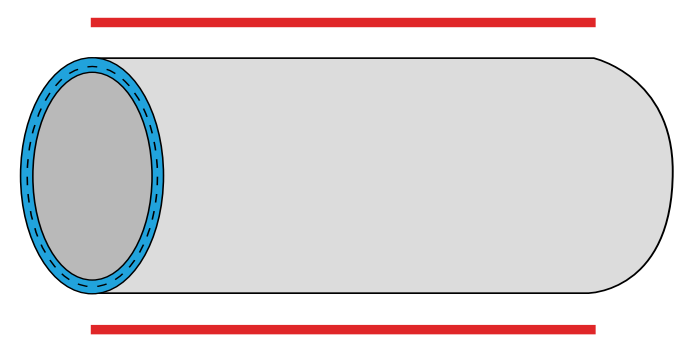

Pure Mem brane Theory

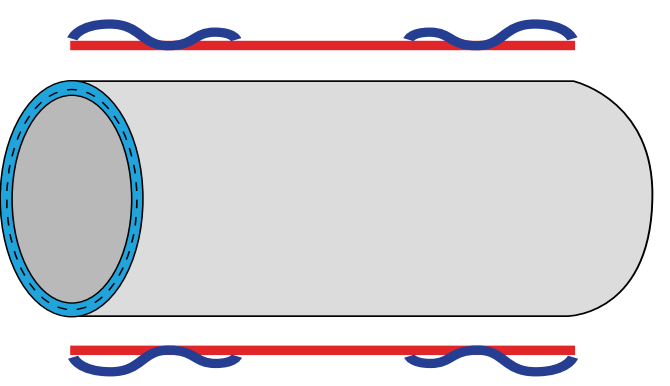

Pseudo -Mem brane Theory

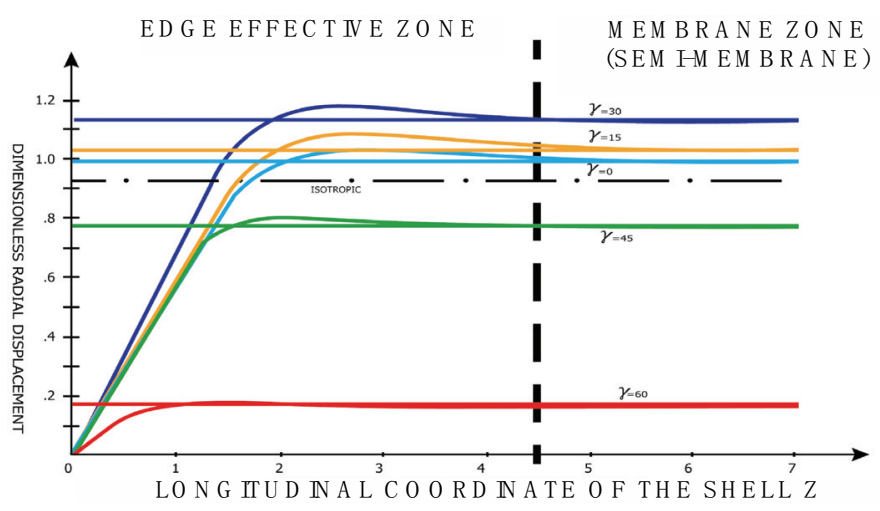

Sem i-M em brane Theory

Fig. 5 Displacement of semi-membrane theory.

$$
\begin{aligned}
& v_{r, y}^{(2)}=0 \\
& v_{z, y}^{(3)}+v_{r, x}^{(0)}=0 \\
& v_{\theta, y}^{(2)}+v_{r, \phi}^{(0)}-v_{\theta}^{(0)}+y v_{\theta, y}^{(0)}=0 \\
& v_{z, x}^{(3)}=s_{11}^{(0)} t_{z}^{(4)}+s_{12}^{(0)} t_{\theta}^{(4)}+s_{11}^{(2)} t_{z}^{(2)}+s_{12}^{(2)} t_{\theta}^{(2)} \\
& v_{\theta, \phi}^{(2)}+v_{r}^{(2)}=s_{21}^{(0)} t_{z}^{(2)}+s_{22}^{(0)} t_{\theta}^{(2)} \\
& v_{\theta, x}^{(2)}+v_{z, \phi}^{(3)}+y v_{\theta, x}^{(0)}=s_{66}^{(0)} t_{\theta z}^{(3)} \\
& t_{r z, y}^{(7)}+\left(y t_{r z}^{(5)}\right), y+t_{\theta z, \phi}^{(5)}+y t_{z, x}^{(2)}+t_{z, x}^{(4)}=0 \\
& t_{r \theta, y}^{(6)}+\left(y t_{r \theta}^{(4)}\right), y+t_{\theta, \phi}^{(4)}+t_{r \theta}^{(4)}+t_{\theta z, x}^{(3)}=0 \\
& t_{r, y}^{(6)}+(y t \underset{r}{(4)})_{, y}+t_{r \theta, \phi}^{(4)}-t_{\theta}^{(4)}=0
\end{aligned}
$$


By integrating the first three equations of the above, we will obtain the following equations:

$$
\begin{aligned}
& v_{r}^{(2)}=v_{r}^{(2)}(x, \phi), \quad v_{z}^{(3)}=v_{z}^{(3)}(x, \phi)-v_{r, x}^{(0)} y \\
& v_{\theta}^{(2)}=v_{\theta}^{(2)}(x, \phi)+\left(v_{\theta}^{(0)}-v_{r, \phi}^{(0)}\right) y
\end{aligned}
$$

Insert the displacements obtained in Eq. (5) into the middle three equations of Eq. (6) to obtain the following equations:

$$
\begin{aligned}
& v_{z, x}^{(3)}-v_{r, x x}^{(0)} y=s_{11}^{(0)} t_{z}^{(4)}+s_{12}^{(0)} t_{\theta}^{(4)}+s_{11}^{(2)} t_{z}^{(2)}+s_{12}^{(2)} t_{\theta}^{(2)} \\
& \left.s_{21}^{(0)} t_{z}^{(2)}+s_{22}^{(0)} t_{\theta}^{(2)}=v_{\theta, \phi}^{(2)}+v_{\theta, \phi}^{(0)}-v_{r, \phi \phi}^{(0)}\right) y+v_{r}^{(2)} \\
& s_{66}^{(0)}{ }_{t}^{(3)}=v_{\theta, x}^{(2)}+v_{z, \phi}^{(3)}+2\left(v_{\theta, x}^{(0)}-v_{r, x \phi}^{(0)}\right) y
\end{aligned}
$$

By combining the equation for $V_{z, x}$ in Eq. (6), we can obtain the in-plane stress-strain relations as follows:

$$
\left\{\begin{array}{c}
t_{z}^{(2)} \\
t_{t}^{(2)} \\
\theta \\
\theta z
\end{array}\right\}=\left[\begin{array}{ll}
(3) \\
\varepsilon^{2}
\end{array}\right\}+\left[\begin{array}{ll}
\varepsilon_{1} \\
\varepsilon_{2}
\end{array}\right\}\left\{\begin{array}{c}
k_{1} \\
k_{2} \\
\varepsilon_{12}
\end{array}\right)
$$

where

$$
\begin{aligned}
& {[C]=\left[\begin{array}{lll}
s_{11}^{(0)} & s_{12}^{(0)} & 0 \\
s_{21}^{(0)} & s_{22}^{(0)} & 0 \\
0 & 0 & s_{66}^{(0)}
\end{array}\right]-1} \\
& \varepsilon_{1}=v_{z, x}^{(1)} \\
& \varepsilon_{2}=v_{\theta, \phi}^{(2)}+v_{r}^{(2)} \\
& \varepsilon_{12}=v_{\theta, x}^{(2)}+v_{z, \phi}^{(3)}
\end{aligned}
$$




$$
\begin{aligned}
& \mathrm{K}_{1}=0 \\
& \mathrm{~K}_{2}=\mathrm{v}_{\theta, \phi}^{(0)}-\mathrm{v}_{\mathrm{r}, \phi \phi}^{(0)} \\
& \mathrm{K}_{12}=2\left(\mathrm{v}_{\theta, \mathrm{x}}^{(0)}-\mathrm{v}_{\mathrm{r}, \mathrm{x} \phi}^{(0)}\right)
\end{aligned}
$$

Substituting $t_{z}, t_{\theta}$ and $t_{\theta z}$ into the first approximation equations of Eq. (5), we will obtain the following transverse stresses:

$$
\begin{aligned}
t_{r z}^{(5)}= & \left.T_{r z}^{(5)}(x, \phi)-\left[\left(V_{\theta, x}^{(2)}+V_{z, \phi \phi}^{(3)}\right) A_{33}+2\left(V_{\theta, x \phi}^{(0)}-V_{r, x}^{(0)}\right)_{33}\right] B_{33}\right] \\
& -\left[V_{z, x x}^{(1)} A_{11}+\left(V_{\theta, x \phi}^{(2)}+V_{r, x}^{(2)}\right) A_{12}+\left(V_{\theta, x \phi}^{(0)}-V_{r, x \phi \phi}^{(0)}\right) B_{12}\right] \\
t_{r \theta}^{(4)}= & T_{r \theta}^{(4)}(x, \phi)-\left[V_{z, x \phi}^{(1)} A_{12}+\left(V_{\theta, \phi \phi}^{(2)}+V_{r, \phi}^{(2)}\right) A_{22}+\left(V_{\theta, \phi \phi}^{(0)}-V_{r, \phi \phi \phi}^{(0)}\right) B_{22}\right] \\
t_{r}^{(4)}= & T_{r}^{(4)}(x, \phi)+\left[V_{z, x}^{(1)} A_{12}+\left(V_{\theta, \phi}^{(2)}+V_{r}^{(2)}\right) A_{22}+\left(V_{\theta, \phi}^{(0)}-V_{r, \phi \phi}^{(0)}\right) B_{12} 1\right.
\end{aligned}
$$

After mathematical manipulation and inserting boundary conditions, we can obtain the following final equations:

$$
\begin{aligned}
& {\left[\underline{\mathrm{D}}_{-12}-\left(\underline{\mathrm{D}}_{22}-_{12}^{\mathrm{A}} / \underline{\mathrm{A}}_{22}\right)\right] \mathrm{v}_{\mathrm{z}, \mathrm{x} \phi \phi}+\left[\underline{\mathrm{A}}_{11}-\left(\underline{\mathrm{A}}_{12}^{2} / \underline{\mathrm{A}}_{22}\right)\right] \mathrm{v}_{\mathrm{z}, \mathrm{xxx}}+\left[\underline{\mathrm{D}}_{12}-\left(\underline{\mathrm{D}}_{22} \underline{\mathrm{A}}_{12} / \underline{\mathrm{A}}_{22}\right)\right] \mathrm{v}_{\mathrm{z}, \mathrm{x} \phi \phi \phi \phi}} \\
& +\left[\underline{\mathrm{E}}_{22}-\left(\underline{\mathrm{B}}_{22} \underline{\mathrm{D}}_{22} / \underline{\mathrm{A}}_{22}\right)\right] \mathrm{V}_{\theta, \phi \phi \phi}+\left[\underline{\mathrm{B}}_{12}-\left(\underline{\mathrm{A}}_{12} \underline{\mathrm{B}}_{22} / \underline{\mathrm{A}}_{22}\right)\right] \mathrm{V}_{\mathrm{e}, \mathrm{xx \phi}}+ \\
& {\left[\underline{\mathrm{E}}_{22}-\left(\underline{\mathrm{B}}_{22} \underline{\mathrm{D}}_{22} / \underline{\mathrm{A}}_{22}\right)\right] \mathrm{V}_{\theta, \phi \phi \phi \phi \phi}+\left[-\underline{B}_{12}+\left(\underline{\mathrm{A}}_{12} \underline{\mathrm{B}}_{22} / \underline{\mathrm{A}}_{22}\right)\right] \mathrm{V}_{\mathrm{r}, \mathrm{xx} \phi}-} \\
& \left.\left[\underline{\mathrm{E}}_{22}-\left(\underline{\mathrm{B}}_{22} \underline{\mathrm{D}}_{22} / \underline{\mathrm{A}}_{22}\right)\right] \mathrm{V}_{\mathrm{r}, \phi \phi \phi \phi}-\left[\underline{\mathrm{E}}_{22}-\underline{\mathrm{B}}_{22} \underline{\mathrm{D}}_{22} / \underline{\mathrm{A}}_{22}\right)\right] \mathrm{V}_{\mathrm{r}, \phi \phi \phi \phi \phi \phi} \\
& \left(\underline{A}_{12} / \underline{A}_{22}\right) \mathrm{p}^{*}, \mathrm{xx}=0
\end{aligned}
$$

where

$$
E_{i j}=\int_{0}^{y} E_{i j} d \rho
$$

The above differential equations are the governing equations of semi-membrane theory.

\section{Conclusions}

First approximation shell theories are derived by use of the method of asymptotic integration of the exact three-dimensional elasticity equations for a non-homogeneous anisotropic circular cylindrical shell. The analysis is valid for materials which are non-homogeneous to the extent that their properties are allowed to vary with the thickness coordinate $(r)$.

The first approximation theory derived in this analysis represents the simplest possible shell theories for the corresponding length scales considered. Although twenty one elastic coefficients are present in 
the original formulation of the problem, only six appear in the first approximation theories. Depending on the length scales, longitudinal and circumferential, we obtain the governing equations and explain the particular behavior of each theory.

For pure-membrane theory, we used both longitudinal and circumferential length scales same as internal radius of the shell. Pseudo-membrane theory is simply a by-product of laminated wall thickness of different anisotropic materials, that is, the difference between geometric half thicknesses and the true mechanical neutral axis.

In the case of semi-membrane theory, we used the longest possible longitudinal length scale together with normal circumferential length scale which is inner radius of the shell. Because of the longest longitudinal length scale, the boundary conditions of the other end of the cylindrical shell become meaningless, which is the particular behavior of semi-membrane theory.

Semi-membrane shell theory is very useful for long cylindrical shells, such as long rocket fuel storage tank and long airplane fuselage structures.

\section{Acknowledgements}

The research was sponsored by Summit Partners in Menlo Park, California, USA, and is graciously acknowledged.

\section{References}

[1] Widera, O. E. 1970. "An Asymptotic Theory for the Motion of Elastic Plates.” Acta Mechanica 9: 54.

[2] Vlasov, V. Z. n.d. "General Theory of Shells and Its Application in Engineering." NASA TT F-99, National Tech. Information Service.

[3] Vlasov, V. S. 1951. "Basic Differential Equations in General Theory of Elastic Shells." NACA Technical Memorandum 1241.

[4] Goldenveiser, A. L. 1961. Theory of Elastic Thin Shells. Oxford: Pergamon Press.
[5] Ting, T. C. T. 1996. Anisotropic Elasticity. Oxford Engineering Science Series 45. Oxford: Oxford University Press.

[6] Reddy, J. N. 2003. Mechanics of Laminated Composite Plates and Shells (2nd ed.). Florida: CRC Press.

[7] Calladine, C. R. 1988. Theory of Shell Structures. Cambridge: Cambridge University Press.

[8] Donnell, L. H. 1976. Beams, Plates and Shells. McGraw-Hill Book Company.

[9] ASME Boiler Code. 2015. Boiler \& Pressure Vessel Code.

[10] Chung, S. W., Hong, S. G., and Mendenhall, D. A. 2017. "Compare and Contrast Bending Shell Theories of Hybrid Anisotropic Materials." International Journal of Composite Materials 7 (1): 8-19.

[11] Chung, S. W., and Park, S. M. 2016. "A Shell Theory of Hybrid Anisotropic Materials." International Journal of Composite Materials 6 (1): 15-25.

[12] Chung, S. W., and Hong, S. G. 2017. "Pseudo Membrane Shell Theory of Hybrid Anisotropic Materials.” Journal of Composite Structures 160 (1): 586-93.

[13] Chung, S. W., and Ju, G. S. 2017. "Semi-membrane Shell Theory of Hybrid Anisotropic Materials." European Journal of Engineering and Technology 5 (2).

[14] Tonabene, F., and Fantuzzi, N. n.d. "Mechanics of Laminated Composite Doubly-Curved Shell Structures." ISBN 978-88-687-6.

[15] Love, A. E. H. 1944. A Treatise on the Mathematical Theory of Elasticity. New York: Dover Edition.

[16] Reissner, E. 1945. "The Effect of Transverse Shear Deformation on the Bending of Elastic Plates." J. Appl. Mech. 12 (2): 69-77.

[17] Johnson, M. W., and Widera, O. E. 1969. "An Asymptotic Dynamic Theory for Cylindrical Shells." Studies Appl. Math. 48: 205.

[18] Birman, V. 1992. "Extension of Vlasov's Semi-membrane Theory to Reinforced Composite Shells." J. Appl Mech 59 (2): 462-4.

[19] ACI 318 Committee. 2011. ACI 318-11: Building Code Requirements for Structural Concrete and Commentary.

[20] Blaauwendraad, J., and Hoefakker, J. H. 2014. Structural Shell Analysis. Berlin, Germany: Springer.

[21] Ambartsumian, S. A. 1991. Fragments of the Theory of Anisotropic Shells. World Scientific Publishing Co.

[22] Chung, S. W., and Ju, G. S. 2018. "Semi-membrane and Effective Length Theory of Hybrid Anisotropic Materials." International Journal of Composite Materials 7 (3): 103-14. 